\title{
SUPERCONDUCTIVITY
}

$\mathrm{T}$ HE Low Temperature Group of the Physical Society was formed shortly after the Second World War, and since then it has regularly held meetings and discussions on topics of interest to users of low-temperature techniques, both industrial and academic. On October 8 a meeting was held in the Science Museum, London, at which four speakers from different universities presented papers concerned with the problem of superconductivity. Prof. D. M. Newitt was in the chair.

Dr. A. B. Pippard (Cambridge) gave a short account of some of the aims of the current work in the Royal Society Mond Laboratory, Cambridge. The multiplicity of mutually exclusive theories which have been advanced to explain the fundamental mechanism of superconductivity is in his opinion evidence that there is not nearly enough known of the experimental behaviour of superconductors, particularly as regards the finer details of their behaviour. One of the aims of the experimenter should be to construct a physical model of superconductivity which should provide a unified account of the various aspects of the phenomenon and as definite as possible a picture of what must be given a fundamental explanation in terms of the electronic structure of metals. Two important contributions to this end are the theory of $F$. and $H$. London of the relation between current and magnetic field, and the two-fluid model of Gorter and Casimir. The former accounts for the almost perfect diamagnetism of a superconductor (Meissner effect), and implies exponential penetration of field to a small depth; the latter correlates the thermodynamic properties with the temperature variation of the depth of penetration. More searching tests of these ideas are now being undertaken. The thermal conductivity and the resistance at very high frequencies both yield information concerning the normal electrons, and the present position seems to be that although the two-fluid model provides a rough explanation of the observations, there is room for improvement, especially as regards the high-frequency resistance. Measurements on thin films and colloidal particles in principle enable the London theory to be tested, but as yet, owing to the extreme delicacy of the experiments, no definite evidence has been obtained. On the other hand, measurements of the absolute magnitude of the penetration depth and of its variation when small amounts of impurity are present suggest that the London theory may be improved by bringing into it the mean free path of the electrons or an analogous parameter. Support for this view comes from studies of the surface energy between normal and superconducting phases, which has been found to be dependent on the mean free path. It is hoped that further work on these lines will eventually permit a better understanding of the nature of the supercurrent and of superconductors in general.

The other speakers discussed more specific topics with which they have been recently concerned. Mr. $H$. Teague (Bristol) gave an account of the properties of the so-called 'hard' superconductors-vanadium, tantalum and niobium-which are typical of superconductors belonging to Groups 4 and 5 of the Periodic Table. In contrast to tin and other 'soft' superconductors, these have rather broad transitions and the Meissner effect is often very imperfect. There is also a marked tendency for the destruction of diamagnetism to occur at a lower magnetic fieldstrength than is required to restore resistance. These effects are likely to be caused by inhomogeneities produced either by mechanical working or by interstitial impurity atoms. The former may be removed by prolonged annealing in vacuo at a very high temperature, but this rarely removes dissolved impurities; indeed, if the vacuum is not good, additional gaseous impurities may go into solution. Wexler and Corak have demonstrated the deterioration in the behaviour of vanadium resulting from interstitial impurities, while Webber finds no such effect in tantalum. It is therefore likely that, in the latter, mechanical strains are the more important influence. The improvement which vacuum annealing produces in niobium has been studied at Bristol, and the observations support the view that both causes of inhomogeneity are important. It has also been found that some samples after annealing behave very strangely : the resistance drops fairly sharply at the transition temperature for niobium, but not to zero, and the drop is less after more prolonged annealing. It seems likely that individual grains become superconducting but the grain boundaries, which are observed to be broadened by annealing, do not. During the discussion Dr. K. Mendelssohn (Oxford) suggested that the grain boundaries might act as nuclei for the growth of a different crystalline modification which is not a superconductor.

Mr. C. A. Renton (Oxford) discussed the thermal conductivity of superconductors, particularly in the temperature-range below $1^{\circ} \mathrm{K}$. According to the two-fluid model of Gorter and Casimir the superconducting electrons have no entropy, and are unable, therefore, to contribute to the conduction of heat, which is effected by the normal electrons and by elastic waves in the ionic lattice. If the scattering of electrons by the lattice is predominantly due to impurities rather than thermal oscillations, the temperature dependence of the electronic contribution is found to agree well with Heisenberg's theory at temperatures not too far below the transition tem. perature. At lower temperatures Koppe's refinement of the theory appears to be substantiated, and the electronic contribution tends to vanish more rapidly than is predicted by Heisenberg's treatment. At the same time, according to a hypothesis of Hulm, the lattice contribution should increase with lowering of the temperature, since only the normal electrons may be expected to be capable of interacting with the lattice and scattering lattice waves. This expectation is confirmed by the observation that at the lowest temperatures the conductivity in lead, tin and indium varies as $T^{3}$, the expected variation for lattice conductivity. The mean free path of the lattice waves is found under these conditions tô be of the order of the diameter of the specimen, so that apparently only boundary scattering of lattice waves is effective. In tantalum, on the other hand, the thermal conductivity below $1^{\circ} \mathrm{K}$. varies as $T^{2}$, which may be accounted for by the hypothesis that there remains even at $0^{\circ} \mathrm{K}$. a residuum of normal electrons, insufficient to contribute noticeably to the conductivity, but adequate to scatter lattice waves. 
Measurements have also been made on the thermal conductivity in the intermediate state, which may be considerably less than in either of the pure superconducting or normal states; the effect is observed in both longitudinal and transverse fields, and indicates additional scattering processes at the phase boundaries. In the course of the discussion it was mentioned that Laredo at Cambridge has obtained by experiments on impure tin powerful support for the view that lattice conductivity is dominant at the lowest temperatures.

Finally, Prof. G. O. Jones (Queen Mary College, London) described work carried out under his direction by Mr. P. F. Chester on superconductivity at high pressures. The transition temperature is affected by volume changes, and a study of the magnitude of the effect is of interest in connexion with fundamental theories, as those of Fröhlich and Bardeen. By means of Bridgman's crossed knife-edge technique, modified for use at helium temperatures, pressures up to 40,000 atmospheres may be applied to small specimens, the transition of which to superconductivity is observed magnetically. The transition temperature of tin is reduced to about $3^{\circ} \mathrm{K}$. from $3.73^{\circ} \mathrm{K}$. by the application of 17,000 atmospheres; and although the transition is not very sharp at the high pressure, it is sharp enough to demonstrate that over the majority of the specimen the pressure is nearly constant. So far as the experiments go, there is no evidence for any change in slope of the criticalfield curve under pressure. In thallium, contrary to a report by Russian workers, at lower pressures the transition temperature is also reduced by pressure, but only by about one-tenth of the amount found in tin. Of considerable interest is bismuth, for a long time believed to be on the verge of being a superconductor. Hilsch found that bismuth films deposited at $4^{\circ} \mathrm{K}$. were superconducting, and it now turns out that massive bismuth is superconducting at pressures greater than 20,000 atmospheres, the transition temperature, $7^{\circ} \mathrm{K}$., being nearly constant up to 41,000 atmospheres. Removal of the pressure destroys superconductivity. Since there is a phase-transition in bismuth at room temperature to a more closepacked structure at about 25,000 atmospheres, it seems natural to suppose that it is this modification which becomes superconducting. Other metals being studied are calcium and strontium, which according to Fröhlich's criterion might be expected to become superconducting, but this is not observed up to 40,000 atmospheres.

A. B. Pippard

\section{EMPIRICAL RESEARCH IN INDUSTRY}

W IDELY varying methods of research for studying the behaviour of individuals and organizations in industry were discussed at an informal week-end gathering held in York during October 2-5 under the auspices of the Acton Society Trust and the York Civic Trust. With backgrounds in the disciplines of anthropology, psychology, sociology, industrial relations, industrial and public administration, operational research, and engineering, twenty-three persons from nineteen university and other research groups met with the common bond that all are actively concerned with research studies of industry.
Two full days of informal discussion were prefaced by a reception, held in the ancient St. Anthony's Hall which now forms part of the Borthwick Institute of Historical Research. The conference was welcomed to York by the chairman of the York Civic Trust, Dr. J. B. Morrell, the chairman of the Acton Society Trust, Prof. A. W. Harvey, and the Lord Mayor of York. On behalf of the visitors, Mr. R. G. Stansfield (Department of Scientific and Industrial Research) said that many research workers in different parts of Great Britain and working in very different ways, are studying what may be called "the natural history of industry"; often these workers are not well aware of the methods, problems and interests-and sometimes even of the existence -of other workers on related problems. The object of the meeting was to bring together some of these people, so that they could get to know each other and stimulate each other's ideas, with plenty of time for free and frank discussion. York, with its tradition of social pioneering, associated with such names as Seebohm Rowntree and William Temple, was a fitting place for such a conference; and especially in view of Rowntree's pioneer empirical studies of poverty in York, and his association with the early days of the Industrial Health Research Board.

On October 3, Mr. Tom Burns (Edinburgh University Social Science Research Centre) spoke of his research into factory organization. He stated that we are largely at the stage of crude ignorance of what management does; he himself had used the Carlson technique to record how managers spend their time. His findings suggest that in some organizations it would be wrong to talk in terms of vertical communication; the flow of communication is much more 'lateral' than top management usually thinks, and vertical communication is better thought of as 'controlled leaks' up and down from the lateral system. Mr. R. Denerley (Medical Research Council Industrial Psychology Research Group) mentioned six investigations by the Group now under way. The methods used are those of general observation, analysis of factory records, and interviewing techniques; he described some of the work done on investigating and validating various interview techniques. Discussion of these papers took up the second session.

In each subsequent session discussion was prefaced by a single, short paper. Mr. H. Clegg (Fellow of Nuffield College, Oxford) described his methods, as a student of industrial relations, in the study of largescale organizations. Mr. J. R. Gass (Department of Scientific and Industrial Research), analysing broadly research problems and methods, pointed out that the three previous papers described the use of very different interviewing methods. Each method is appropriate in its own place; his criticism was that the users are unwilling to state the limitations of their method. If between the 'human relations' and 'industrial relations' schools a conflict-a false conflict-seems to be replacing mutual indifference, it is a sign of the impact of mutual relevance of the two approaches. At present, each can be criticized for what it ignores; much American sociology on the changing of habits ignores economic forces and the effect of large-scale organizations external to the factory; the industrial relations school, which studies these large-scale organizations, is interested in the conflicts of power, but tends to ignore the informal structure and personal forces. The link between the two approaches may be found in the 